\title{
Research
}

\section{Photovoltaic Module Reliability Model Based on Field Degradation Studies}

\author{
Manuel Vázquez ${ }^{1 *, \dagger}$ and Ignacio Rey-Stolle ${ }^{2}$ \\ ${ }^{1}$ EUITT, Instituto de Energía Solar-UPM, Madrid, Spain \\ ${ }^{2}$ ETSIT, Instituto de Energía Solar-UPM, Madrid, Spain
}

Crystalline silicon photovoltaic (PV) modules are often stated as being the most reliable element in PV systems. This presumable high reliability is reflected by their long power warranty periods. In agreement with these long warranty times, PV modules have a very low total number of returns, the exceptions usually being the result of catastrophic failures. Up to now, failures resulting from degradation are not typically taken into consideration because of the difficulties in measuring the power of an individual module in a system. However, lasting recent years $P V$ systems are changing from small isolated systems to large grid-connected power stations. In this new scenario, customers will become more sensitive to power losses and the need for a reliability model based on degradation may become of utmost importance. In this paper, a PV module reliability model based on degradation studies is presented. The main analytical functions of reliability engineering are evaluated using this model and applied to a practical case, based on state-of-the-art parameters of crystalline silicon PV technology. Relevant and defensible power warranties and other reliability data are obtained with this model based on measured degradation rates and time-dependent power variability. In the derivation of the model some assumptions are made about the future behaviour of the products-i.e. linear degradation rates-although the approach can be used for other assumed functional profiles as well. The method documented in this paper explicitly shows manufacturers how to make reasonable and sensible warranty projections. Copyright (C) 2008 John Wiley \& Sons, Ltd.

KEY WORDS: photovoltaic module; reliability; degradation; MTTF; warranty

Received 24 July 2007; Revised 9 January 2008

\section{INTRODUCTION}

Photovoltaic (PV) modules are often considered as the most reliable element in PV systems. ${ }^{1}$ However, PV module reliability data are not shown on commercial datasheets in the same way as it is with other products

* Correspondence to: Manuel Vázquez, Electrónica Física, EUIT de Telecomunicación, Campus Sur-UPM, Km 7 Carretera de Valencia, 28031 Madrid, Spain.

†E-mail: vazquez@euitt.upm.es such as electronic devices and electric power supplies. Conversely, the high reliabilities associated to PV modules are indirectly reflected in the output power warranties usually provided in this industry, which are currently in the range of 25 years, and may reach 30 years in a near future. ${ }^{2,3}$ As a matter of fact, PV modules have a very low total number of returns, the exceptions usually being the result of catastrophic failures, in agreement with this long warranty on output power. Nevertheless, it is also widely known 
that PV module performance when deployed outdoors decreases steadily over time. ${ }^{2}$ After several years of operation this decrease will affect PV module reliability.

Reliability evaluation based on degradation models ${ }^{4}$ is commonly applied in highly reliable products as a cost effective and confident way of evaluating their reliability. In this paper a degradation model for PV modules is presented and subsequently applied in the quantitative analysis of PV module reliability. With this model the different parameters related to module reliability such as the reliability function, failure rate function, the Mean time to failure (MTTF) or the warranty period can be assessed based on PV module degradation in the field.

This paper is divided into four core sections, plus this introduction and the final conclusions. The different aspects of solar module warranty and qualification are briefly revisited and summarized in Section 'Product Warranty and Qualification Tests'; PV module degradation data from different published studies are reviewed in Section 'Review of Field Degradation Studies of PV Modules'; the mathematical foundations of the model developed are presented in Section 'Model Development' and the reliability model is applied to the available PV module data in Section 'Model Application', and the main reliability parameters of the technology are calculated.

\section{PRODUCT WARRANTY AND QUALIFICATION TESTS}

It is often claimed that PV modules are the most reliable element in PV systems. This high reliability is currently reflected in different aspects such as the long warranties on output power provided by virtually all manufacturers of crystalline (either mono or poly) silicon PV modules and the existence of strict qualification standards that the PV industry follows systematically. In the following subsections, we will revisit the concepts of PV warranties and qualification standards to clarify their links to reliability analysis, which are sometimes not so straightforward.

\section{Photovoltaic module warranties}

A PV module warranty usually refers to two different items: a warranty for materials and workmanship, which usually ranges from 1 to 5 years, and a warranty on the power produced by the PV module. This second warranty typically guaranties that after the first 10-12 years the output power of the module will be at least $90 \%$ of its initial nominal power and that after 20-25 years of operation the output power of the module will be at least $80 \%$ of its initial nominal power. In addition, the small print of the warranty information sometimes contains two extra boundary conditions associated to the manufacturing tolerance for the module power and the measurement tolerance. In recent years manufacturers have redefined the concept of output power warranty ${ }^{5}$ which is now referred to as the minimum nominal power taking into account module power tolerances, and not with respect to the nominal power. The measurement tolerance is also quantified as an extra $3 \%$ of uncertainty. For instance, applying the terms of the typical power warranty given at the beginning of this paragraph to a PV module with a nominal power of $P_{0}=100 W_{p}$ with a $5 \%$ tolerance module power (very common in current PV modules) and the aforementioned 3\% measurement tolerance, then the minimum final power after 25 years of operation would be:

$$
P_{\text {limit }}=P_{0} \times 0.95 \times 0.8 \times 0.97=73.7 \mathrm{~W}
$$

In other words, by taking into account the extra tolerances included in the warranty conditions the initial $80 \%$ of the nominal power eventually relaxes to an effective $73.7 \%$. Of course, the change in the way the final power is calculated (considering various tolerances or adding other constraints) maybe a fast way for a manufacturer to keep the number of module returns low, but it does not inherently improve the product reliability and thereby is not a good long-term strategy in the quest for longer power warranties.

The average PV module power warranty has increased from 5 years (typical values before 1987) to 25 years (typical values since 1999). ${ }^{2,3}$ It is worth mentioning that a 25 or 30 -year long warranty is a remarkably high value when compared to the warranties of many other commercial products produced by the electronics industry. Obviously, these warranties are not the result of thorough tests in the field simply because the modules have not been on the market long enough. They are probably the combined outcome of empirical approaches, field tests of limited duration and a set of particular qualification/ degradation tests that each manufacturer might have developed for this purpose. Despite its phenomenological origins, the power warranties provided nowadays by the PV module industry are reasonable, 
from the manufacturer's point of view, given the low (on average) number of returned modules. Nevertheless, it should be noted that currently the majority of the returned modules have suffered catastrophic failures. Only few returns are the result of a careful monitoring by the customer of the power loss of the modules during their operating life, simply because it is very difficult to measure the power of an individual module within the PV system and, in addition, power losses are frequently attributed to causes other than degradation (dirt, measurement tolerances, etc.). However, PV systems are moving from small isolated systems to large AC grid-connected systems with favourable feed-in tariffs. In this new scenario, the sensitivity of customers to power losses over time (i.e. to PV module degradation) is definitely changing. We strongly believe that in this new scenario the aid of reliability models based on degradation may become of utmost importance as a tool to quantify reliability parameters and thereby to establish realistic (for the customer) and defensible (for the manufacturer) power warranties.

\section{Photovoltaic module qualification standard}

While most PV module manufacturers carry out qualification tests on their modules, there has been little reliability testing in the industry., ${ }^{2,3}$ A qualification test is a set of defined experiments with pass/fail criteria and no quantification. Reliability tests are designed to evaluate failures, to quantify them and to help understand the failure mechanisms in order to improve the reliability of a given product. Consequently, a reliability test goes beyond qualification tests in order to bring about the failure using essentially two different strategies: either applying stresses for longer exposure times or by applying higher stress levels for shorter exposure times. In reliability tests, when (reliability quantification), what and why (how to avoid the main failure sources) the failure occurs is analysed.

Most commercial crystalline silicon PV module manufactures qualify their modules with standard IEC 61215 (crystalline silicon terrestrial PV modulesdesign qualification and type approval). ${ }^{6}$ This standard was published in 1993 in its first edition and 2005 in a second edition and is based on its direct predecessor, CEC specification 503. ${ }^{7}$ We will describe IEC 61215 briefly just to give the reader an idea of the complexity of the process. This standard requires eight randomly chosen modules:
1. One module is taken as reference and is not subjected to any accelerated stress tests.

2. Another module is characterized electrically (performance at different radiation conditions, bypass diode thermal test and hot spot endurance).

3. The other six modules are divided into three groups of two and subjected to three different mechanical and environmental tests, namely:

- Ultraviolet exposure, 50 thermal cycles and 10 humidity freeze cycles.

- 200 thermal cycles.

- $1000 \mathrm{~h}$ of damp heat, wet leakage current test and mechanical tests.

The modules are evaluated at different stages of the test in order to detect five different defects, which are defined as module failure. These defects are:

1. Visible defects such as cracked cells, bubbles, delamination or loss of mechanical integrity.

2. Open-circuit or ground faults that are monitored both during and at the end of the different tests.

3. Performance losses. The degradation at maximum power must neither exceed the prescribed limit after each test nor $8 \%$ after each test sequence.

4. Insulation test. $1000 \mathrm{~V}$ plus twice the anticipated system voltage is applied to the modules for the insulation test.

5. The wet leakage current test requirements are met at the beginning and the end of each sequence and after the damp heat test.

If all the modules pass the tests, the module type meets the qualification standard. If two or more modules do not pass the tests, the module type does not meet the qualification standard. If a single module fails any test, then two new modules of the same type are subjected to a repeat of the test step which brought about the failure. Qualification occurs only if both modules pass this repetition of the test.

The links of the IEC 61215 qualification test with reliability analysis can be summarized as follows:

- IEC 61215 tests are not reliability tests but can indeed provide some information on reliability. There are several studies that have stated that successfully passing IEC 61215 guaranties between 15 and 20 years of PV module lifetime in a fairly moderate climate. ${ }^{2,8}$ These values are significantly lower than the power warranties for current PV modules and the main manufacturers ${ }^{2,3,9}$ have 
additional in-house tests that must be passed in order to provide greater assurance in providing a 25 -year long power warranty.

- The defects that appear in these IEC 61215 tests are different than those that appear in the field. ${ }^{10,11}$ For instance, the thermal cycles and damp heat tests made using IEC 61215 bring about tedlar delamination rather than tedlar detachment, which is more commonly observed in modules that have degraded under real operating conditions. Accordingly defect analyses (and subsequent correction) on these failures do not necessarily increase the reliability of the product.

- The full process to certify a crystalline silicon module using IEC 61215 costs around 25000 Euros. ${ }^{12}$ Because of these high costs there are many modules from small or medium-size PV manufacturing companies without any certification. The Quality Program for PVs (QuaP-PV) of the World Bank $^{13}$ proposes to reduce standardization costs in order to increase the quality of PV modules.

In summary, having a very established qualification test poses the problem that most PV module manufacturers carry out qualification tests but almost no reliability tests. ${ }^{2}$ These tests are costly and do not provide the overall picture needed for reliability analyses. Because of all these issues we propose a degradation model as a complementary and inexpensive way to evaluate PV module reliability.

\section{REVIEW OF FIELD DEGRADATION STUDIES OF PV MODULES}

In this section, we will evaluate the PV module degradation and reliability data from the literature by taking into account different parameters such as:

- Technology. In this article we will focus on both monocrystalline (c-Si) and polycrystalline silicon (p-Si) PV modules because they are the prevalent technology ${ }^{14}$ in the market (more than $90 \%$ ). Moreover, crystalline silicon modules are also much more reliable ${ }^{15,16}$ than amorphous $\mathrm{Si}$ and CIS (copper indium di-selenide) solar modules.

- Location (temperature, humidity and radiation). PV module lifetime, like in other devices, depends on different ambient conditions such as temperature, humidity or radiation.
PV modules can degrade their performance as a result of different factors ${ }^{17}$ such as:

- Degradation of packaging materials

- loss of adhesion of encapsulants

- degradation of cell/module interconnection

- degradation caused by moisture intrusion

- degradation of the semiconductor device.

In the case of crystalline silicon PV modules, the degradation of the semiconductor is not important because of the stability of the semiconductor material. Field experience indicates that the primary causes of performance losses are associated with mechanisms external to the cell itself such as solder joints, encapsulant browning, delamination and interconnection problems. ${ }^{17}$

There are a number of studies of PV module degradation in the field and some of them are summarized in Table I. The main conclusion of these studies is that solar modules do not usually fail in a catastrophic way but experience a steady power degradation over time. ${ }^{5,15-26}$ This degradation process has been reported to undergo two different stages: within the first year of exposure solar modules exhibit a rapid degradation $(1-3 \%),{ }^{18,19,27}$ thereafter a slower linear degradation rate is observed $(0 \cdot 5-1 \cdot 0 \% /$ year $)$. Another important issue that affects PV module performance is that the variability of PV module power increases over time. ${ }^{18,20,21}$ In the following we will summarize the most significant data from the degradation studies in Table I:

1. The Australian Cooperative Research Center ${ }^{15}$ evaluated the performance of five different PV module technologies- $-\mathrm{C}-\mathrm{Si}, \mathrm{p}-\mathrm{Si}$, triple junction a-Si, CIS and the laser grooved buried contact (LGBC) $\mathrm{c}-\mathrm{Si}$ - over 16 months of operation in a temperate climate (Perth, Australia). The PV modules were installed in 2000 and evident power reduction differences could be measured. Crystalline and polycrystalline silicon PV modules showed a yearly power reduction of $2 \%$ while amorphous and CIS solar modules exhibited a significantly higher power reduction. The results for $\mathrm{c}-\mathrm{Si}$ and p-Si PV modules cannot be used to extrapolate the module lifetime since it is known that the degradation rate is faster during the first year of field operation (i.e. $75 \%$ of the duration of this study). 
Table I. Summary of some studies on PV module field degradation around the world

\begin{tabular}{|c|c|c|c|c|c|}
\hline $\begin{array}{l}\text { Number in Section } \\
\text { 'Review of Field } \\
\text { Degradation Studies of } \\
\text { PV modules'/Reference }\end{array}$ & Location & Test duration & $\begin{array}{l}\text { Module } \\
\text { Tech. }\end{array}$ & $\begin{array}{c}\text { Degradation } \\
\text { rate }(\% / \text { year })\end{array}$ & Comments \\
\hline $1 /[15]$ & $\begin{array}{l}\text { Perth (Australia) } \\
\text { Temperate climate }\end{array}$ & 16-19 months & $\begin{array}{l}\mathrm{c}-\mathrm{Si} \\
\mathrm{p}-\mathrm{Si} \\
\mathrm{a}-\mathrm{Si} \\
\mathrm{CIS}\end{array}$ & $\begin{array}{c}0 \cdot 5-2 \cdot 7 \\
1 \cdot 0-2 \cdot 9 \\
18 \cdot 8 \\
12 \cdot 6\end{array}$ & \\
\hline $2 /[22]$ & $\begin{array}{l}\text { Mesa, Arizona (USA) } \\
\text { Desert climate }\end{array}$ & $\begin{array}{c}2 \cdot 4-4 \text { years } \\
2 \cdot 4-2 \cdot 7 \text { years } \\
2 \cdot 7-6 \cdot 7 \text { years }\end{array}$ & $\begin{array}{l}\mathrm{c}-\mathrm{Si} \\
\mathrm{p}-\mathrm{Si} \\
\mathrm{a}-\mathrm{Si}\end{array}$ & $\begin{array}{c}0.4 \\
0.53 \\
1.16(6 \cdot 7 \text { year }) \text { to } \\
3.52(2.7 \text { year })\end{array}$ & $\begin{array}{l}\text { Initial scattering of the } \\
\text { performance is high }\end{array}$ \\
\hline $3 /[20]$ & $\begin{array}{l}\text { Trinidad, California (USA) } \\
\text { Cool coastal climate }\end{array}$ & 11 years & $\mathrm{c}-\mathrm{Si}$ & $0 \cdot 4$ & $\begin{array}{l}\text { Variability in maximum } \\
\text { power increase } \\
\text { significantly over time }\end{array}$ \\
\hline 4/[18] & $\begin{array}{l}\text { Hamamatsu (Japan) } \\
\text { Temperate climate }\end{array}$ & 10 years & $\mathrm{c}-\mathrm{Si}$ & 0.62 & \\
\hline $5 /[23]$ & $\begin{array}{l}\text { Golden, Colorado (USA) } \\
\text { Mountain continental } \\
\text { climate }\end{array}$ & 8 years & $\mathrm{c}-\mathrm{Si}$ & 0.75 & \\
\hline \multirow[t]{2}{*}{$6 /[24]$} & $\begin{array}{l}\text { Ispra (Italy) } \\
\text { Temperate climate }\end{array}$ & 22 years & $\mathrm{p}-\mathrm{Si}$ & $0 \cdot 3$ (Silicone) & $\begin{array}{l}\text { Two technologies were } \\
\text { evaluated for the } \\
\text { encapsulant, namely } \\
\text { silicone and EVA }\end{array}$ \\
\hline & & & $\mathrm{c}-\mathrm{Si}$ & 0.67 (EVA) & \\
\hline $7 /[28]$ & $\begin{array}{l}\text { Lugano (Switzeland) } \\
\text { Temperate climate }\end{array}$ & 20 years & $\mathrm{c}-\mathrm{Si}$ & $0 \cdot 53$ & $\begin{array}{l}\text { Power variability has } \\
\text { increased significantly }\end{array}$ \\
\hline $8 /[26]$ & $\begin{array}{l}\text { Negev desert (Israel) } \\
\text { Desert climate }\end{array}$ & 3.4 years & $\mathrm{p}-\mathrm{Si}$ & $1 \cdot 3$ & $\begin{array}{l}\text { Tests were performed } \\
\text { under concentrated } \\
\text { light using mirrors } \\
(2.56 \text { ratio) }\end{array}$ \\
\hline
\end{tabular}

c-Si: Crystalline silicon; p-Si: polycrystalline silicon; a-Si: amorphous silicon.

2. In a study carried out by the Arizona State University East Photovoltaic Testing Laboratories, ${ }^{22} 44$ modules from three different technologies were evaluated in Mesa, Arizona in hot-arid climatic conditions. Monocrystalline and polycrystalline silicon modules exhibited virtually the same low power degradation $(\sim 0.5 \%$ yearly power degradation for both $\mathrm{c}-\mathrm{Si}$ and $\mathrm{p}-\mathrm{Si}$ ) while a-Si multijunction modules degraded more intensively ( $1.16 \%$ yearly degradation). There were no significant differences in power degradation in this location with respect to more temperate climate locations.

3. In 1990, the Schatz Energy Research Center $\left(\right.$ SERC) ${ }^{20}$ installed a PV array made up of 192 c-Si PV modules in Trinidad, California. The PV modules worked for 11 years with an overall $4.4 \%$ average power reduction. The variability in maximum power within the modules increased significantly and after 11 years the standard deviation of the module power distribution was twice the initial standard deviation. This increase in variability will severely affect the PV module reliability as will be analysed in the reliability model.

4. A study developed by the Japan Quality Assurance Organization and Solar Techno-Center, ${ }^{18}$ focused on the power degradation of crystalline silicon PV modules manufactured in Japan from 1990 to 1992 and operated outdoors for 10 years in Hamamatsu (Japan). After 10 years, the average power reduction was $6.2 \%$; but $10 \%$ of the PV modules had a power reduction greater than $10 \%$. 
5. A $1 \%$ yearly degradation was reported in c-Si PV modules installed in Colorado ${ }^{23}$ in 1994 and evaluated 8 years later.

6. The Institute for Environment and Sustainability ${ }^{24}$ analysed the performance of 40 silicon (poly and mono) crystalline PV modules that had been working for 20-22 years in Ispra (Italy). The average power degradation of these PV modules depended a lot on the encapsulation. Modules encapsulated with silicone had a $6.4 \%$ average power degradation while modules encapsulated with EVA and a Tedlar aluminium back sheet had a $14.8 \%$ mean power degradation.

7. The MTBF Project ${ }^{28}$ evaluated the performance after 20 years of operation of a $10 \mathrm{~kW}$ PV system installed in Lugano (Switzerland). The modules were made using crystalline silicon technology and after 20 years a $10.5 \%$ average power degradation was measured. Fifty-nine per cent of these modules exhibited a power reduction of less than $10 \%, 35 \%$ of the modules exhibited a power reduction of between 10 and $20 \%$ and only for the remaining $6 \%$ of the modules the power reduction was greater than $20 \%$. An increase in power variability over time was also corroborated in this study.

8. Another interesting work was carried out at the Negev Desert (Israel) ${ }^{26}$ studying the performance of 60 polycrystalline silicon PV modules. The modules had a solar concentration system based on mirrors which increased the irradiance up to 2.56 Suns and were working from July 1992 to December 1995. Associated to the concentration increase, a temperature boost should also be expected-as compared to the temperature of those same modules at that same location without the mirrors - though the authors of this study do not report information on this topic. The measured module power fitted a normal distribution with an average yearly power reduction of $1.3 \%$ and a slight increase in module power variability. Some measurements performed on a limited set of modules installed at that same location without mirrors yielded very similar results.

The main conclusions of all these degradation studies show that:

- When deployed outdoors, the power of PV modules suffers a linear degradation rate over time. The magnitude of this degradation depends on the module technology. The degradation rate for crystalline silicon modules is significantly smaller than that for amorphous Si and CIS modules. Taking crystalline silicon PV modules as a reference, the yearly linear power reduction rate varies from $0.3 \%{ }^{24}$ to more than $1 \% .{ }^{15}$ However, PV module technology is improving continuously ${ }^{25}$ and solar modules manufactured in recent years are expected to have a lower power reduction than solar modules manufactured in the 1980s, which is the case for most modules involved in the studies detailed in Table I.

- PV module power follows a normal distribution as do many other products. It is known from several sources that power variability increases over time but there is little information on how the standard deviation increases over time. The scarce data available vary from a $10 \%$ increase in standard deviation in 3.5 years $^{26}$ up to a $100 \%$ increase in 11 years. $^{20}$ The lack of this kind of study results from the fact that the evolution of the power standard deviation has not been considered as important as average power degradation from a reliability point of view. In this study we will show the importance of this parameter.

\section{MODEL DEVELOPMENT}

In this section, we derive a PV solar module degradation model intended to be a valuable tool for predicting reliability. Reliability prediction based on degradation models is an efficient method for estimating the reliability of highly reliable devices when the observation of failures is rare., ${ }^{4-32}$ Degradation models are more efficient than accelerated tests with few failures. We will obtain analytical expressions for the reliability function, the failure probability density function, the instantaneous failure rate function, the MTTF, and some suggestions on establishing reasonable power warranties, as well as other statistical parameters.

\section{Derivation of the PV module power probability density function}

In order to develop this method we will assume the following hypotheses:

- PV module power will be the reference parameter to evaluate module performance. PV module warranties also use this parameter as a reference. 
- PV module failure will be defined with reference to its nominal or initial power. If, at a given point, the power produced by a PV module is lower than a predefined value (hereinafter $P_{\text {limit }}$ ) then the module will be considered to have failed. As discussed in Subsection 'Photovoltaic module Warranties', $P_{\text {limit }}$ is often defined as a given percentage of the module minimum nominal power.

- PV module power follows a Gaussian distribution. $^{20,33,34}$ Accordingly, the associated probability density function is

$$
p(P)=\frac{1}{\sqrt{2 \pi} \sigma} \exp -\frac{1}{2}\left[\frac{P-\mu}{\sigma}\right]^{2}
$$

where $P$ denotes the module power (in $W_{p}$ ), $\mu$ is its average value (in $W_{p}$ ) and $\sigma$ its standard deviation (in $\left.W_{p}\right)$.

- The average power of the modules $(\mu)$ decreases linearly over time. ${ }^{19,22,23,35}$ In this case $\mu$ can be calculated as

$$
\mu(t)=P_{0}-A t
$$

where $P_{0}$ is the average power in $W_{p}$ at $t=0$ (i.e. the nominal power of the module), $A$ is a parameter that reflects the yearly decrease in module power in $W_{p} /$ year and $t$ is the time in years. Obviously, the validity of Equation 3 is limited to times of less than $P_{0} / A$. Another possible limitation of Equation 3 comes from the assumption that $A$ is a constant over time. For instance in Reference 2, an increasing yearly degradation rate is observed in some accelerated tests. However, none of the studies in Table I report such behaviour and thus we have decided to keep $A$ as a constant. Further research on this topic is needed.

Using Equation 3 it is straightforward to demonstrate that for any pair of successive years the module power decays at a fixed percentage of its initial power:

$$
\frac{\mu(n)-\mu(n-1)}{P_{0}}=-\frac{A}{P_{0}}=\text { constant }
$$

The ratio $A / P_{0}$ is a parameter in inverse time units $\left(\right.$ year $^{-1}$ ) that will hereinafter be referred to as the yearly degradation percentage.

Several authors argue that the limited experimental evidence available is not enough to take the linear degradation rate for granted and suggest that an exponential degradation rate (Equation 5) could be a more suitable trend as is the case in some optoelec- tronic devices: ${ }^{36,37}$

$$
\mu(t)=P_{0} e^{-\alpha t}
$$

In order to not increase the complexity of the discussions we will not consider the exponential degradation rate here. However, it should be noted that both trends exhibit a very similar evolution during the first $10-15$ years - if similar initial yearly degradation rates are assumed-and then the linear degradation rate is a more pessimistic estimate, as reflected in Figure 1.

- The second time dependence of the PV module power probability density function is that of its standard deviation $(\sigma)$. Different authors ${ }^{20,21,26,33}$ have reported that $\sigma$ increases over time in PV modules just as it does in other optoelectronic devices. However there is little information about how standard deviation evolves over time. In a first approach we will assume that standard deviation follows a linear degradation rate over time: ${ }^{4}$

$$
\sigma(t)=\sigma_{0}+B t
$$

$\sigma_{0}$ being the standard deviation at $t=0, B$ a parameter that reflects the yearly increase in standard deviation in $W_{p} /$ year and $t$ is time in years. In this model, the value of parameter $B$ must be limited to a third of that of parameter $A$ in order to prevent the power of the PV modules from varying over time without any physical sense (i.e. if $B>A / 3$ the power of some PV modules increases over time).As with Equation 4, it can be similarly argued that for any two consecutive years the

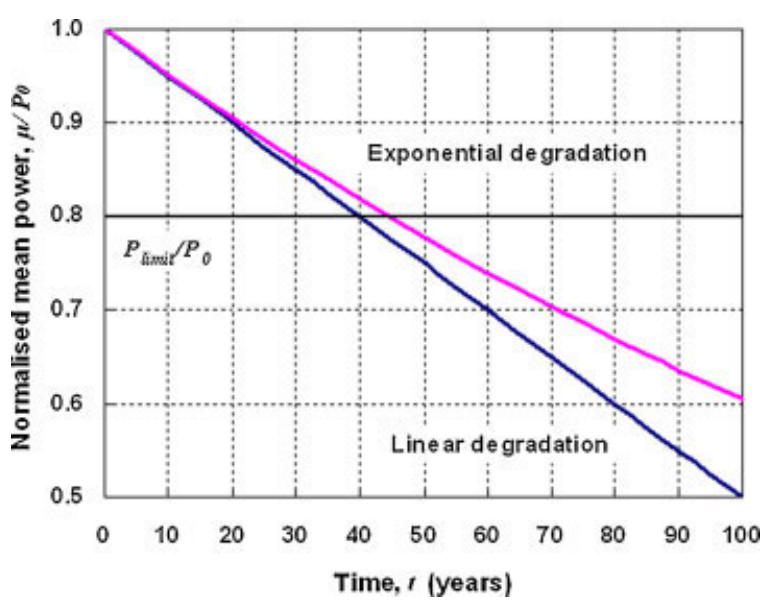

Figure 1. Linear and exponential power degradation rates for a $0.5 \%$ initial yearly degradation

Prog. Photovolt: Res. Appl. 2008; 16:419-433

DOI: $10.1002 / \mathrm{pip}$ 
standard deviation always increases a given percentage of its initial value:

$$
\frac{\sigma(n)-\sigma(n-1)}{P_{0}}=\frac{B}{P_{0}}=\text { constant }
$$

where the ratio $B / P_{0}$ reflects the percentage of the initial power that the standard deviation of the power distribution increases every year.

Taking into account the time variations reflected by Equations 4 and 6, the PV module power at a defined instant will fit the following distribution:

$p(P, t)=\frac{1}{\sqrt{2 \pi}\left(\sigma_{0}+B t\right)} \exp \left[-\frac{1}{2}\left[\frac{P-\left(P_{0}-A t\right)}{\sigma_{0}+B t}\right]^{2}\right]$

Figure 2 aims to clarify the time evolution condensed in Equation 8. In that figure, the normalized PV module power probability density function has been represented at five different times. Two additional lines are also plotted: a horizontal line corresponding to the power limit $\left(P_{\text {limit }}\right)$ and a linearly decreasing line reflecting the evolution over time of the mean module power (i.e. $\mu / P_{0}$ ). At $t=0$ (i.e. for the leftmost curve) the power is distributed around the nominal power of the modules $\left(P / P_{0}=1\right)$ with a small standard deviation. Accordingly, for this case the probability that the power of a PV module is lower than $P_{\text {limit }}$ is almost zero. For the time labelled $t_{50}$ (i.e. for the rightmost curve in Figure 2) the mean value of the

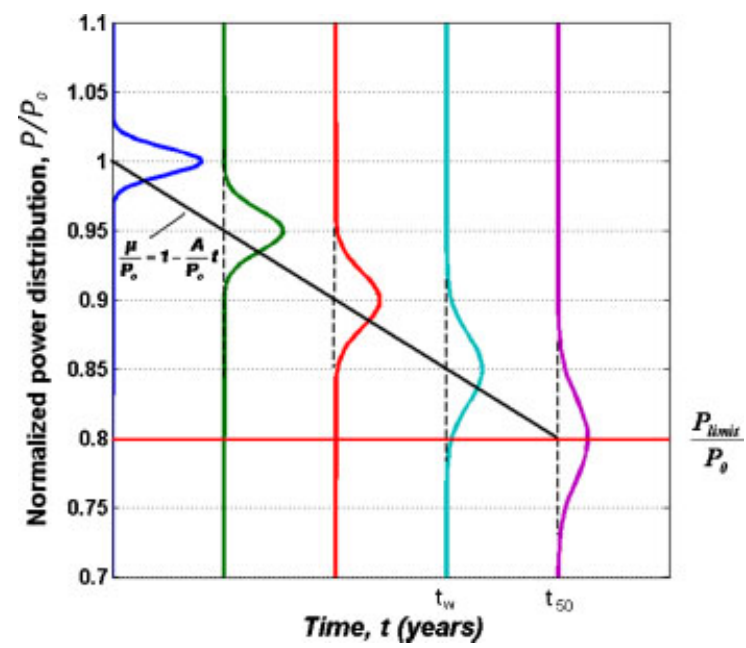

Figure 2. Qualitative representation of the evolution over time of the module power probability density function power distribution coincides with the power limit. Thus, at this point half of the modules included in the distribution have powers lower than $P_{\text {limit }}$ and consequently have failed (i.e. $50 \%$ failure or $50 \%$ reliability). This reliability is too low for any commercial product and therefore this time should occur much later than the period associated to the power warranty. This power warranty period could correspond to the instant noted $t_{\mathrm{W}}$ in Figure 2. At that precise moment only the end of the tail of the module power probability density function is below the power limit line, indicating that only a few modules have failed.

\section{Main reliability functions}

In terms of classic statistical reliability analysis, the reliability function $-R(t)$ - is a time-dependent function that gives the probability of an item operating for a certain amount of time without failure. In the case of a batch of PV modules, the reliability function would give the probability that the power of a PV module in that batch is, at a given time, higher than a power limit that corresponds to failure definition $\left(P_{\text {limit }}\right)$. According to this definition, $R(t)$ can be readily calculated by integrating the PV module power probability density function:

$$
R(t)=\int_{P_{\text {limit }}}^{\infty} p(P, t) \mathrm{d} P=1-\Phi\left(\frac{P_{\text {lim it }}-\left(P_{0}-A t\right)}{\sigma_{0}+B t}\right)
$$

where $\Phi$ is the cumulative probability function for the Gaussian distribution. Using Equation 9 we can evaluate the reliability of a PV module at any time as a function of the different parameters involved, namely $P_{\text {limit }}, P_{0}, A, B$ and $\sigma_{0}$.

The typical shape of the function $R(t)$ can be seen in Figures 3 and 4 . As for any reliability function it equals 1 for $t=0$ and tends towards zero for arbitrarily long times. The transition occurs earlier the higher the $A$ parameter and its roll off is steeper the lower the $B$ parameter.

The probability density function associated to the random variable time to failure is called failure probability density function- $f(t)$ - and is evaluated as

$$
f(t)=-\frac{\mathrm{d} R(t)}{\mathrm{d} t}
$$




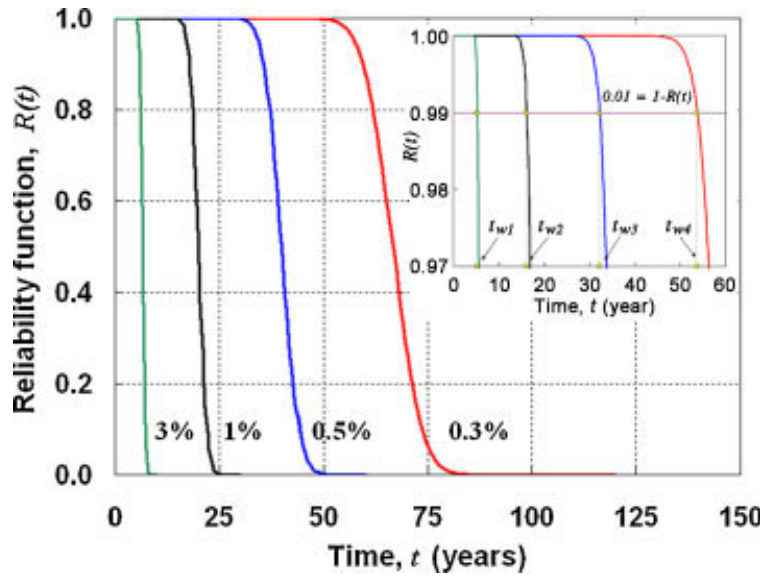

Figure 3. Reliability function for different yearly degradations rates and $B=0$. A zoomed view of the curves for $R>0.95$ is included as an inset

The shape of the function $f(t)$ varies from a Gaussian (if $B=0$, see Figure 5) to asymmetric probability density functions (if $B>0$, see Figure 6). However, in most cases $f(t)$ can be fitted using the Weibull distribution, which is a very versatile probability density functions that can simulate many useful distributions in reliability analyses such as Gaussian and exponential. ${ }^{38}$

Finally, the instantaneous failure rate function$\lambda(t)$-enables the determination of the number of failures occurring per unit time. Consequently, in our case, it will determine the number of failing PV modules per year, among surviving units. The function

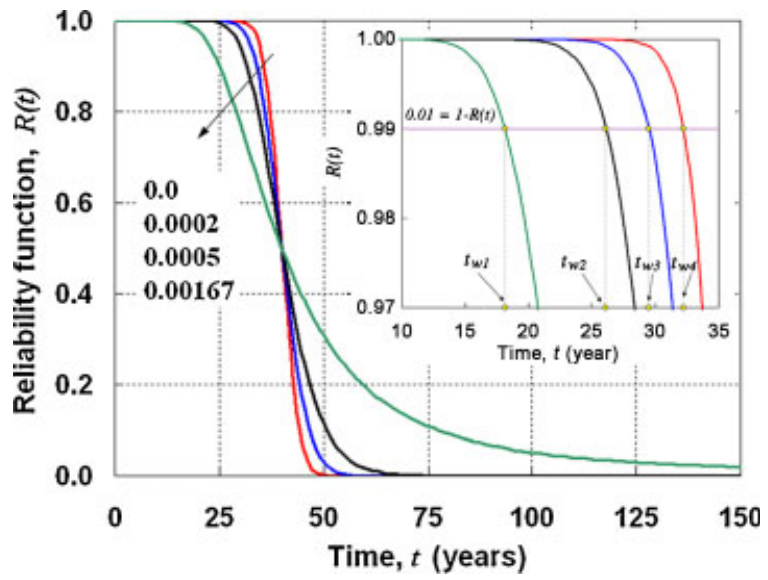

Figure 4. Reliability function for different $B / P_{0}$ values for a fixed $0.5 \%$ yearly degradation rate. A zoomed view of the curves for $R>0.95$ is included as an inset

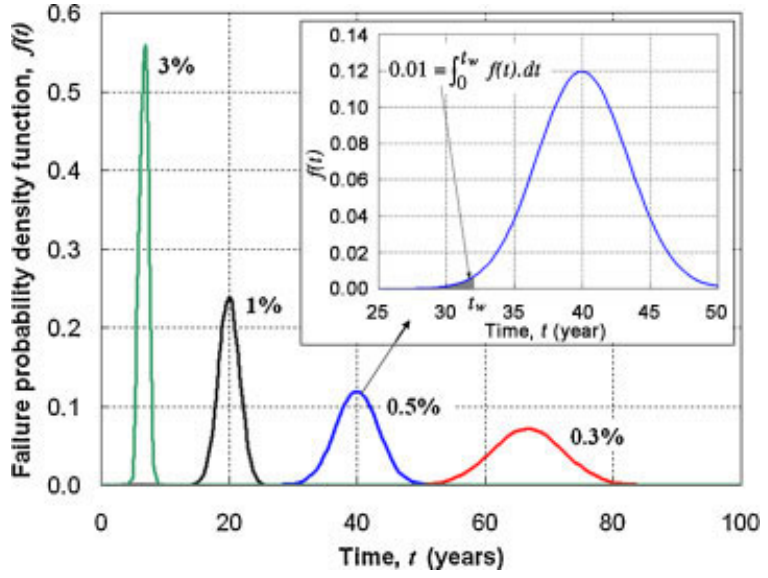

Figure 5. Failure probability density function for different yearly degradation rates and $B=0$. A zoomed view for the case of a $0.5 \%$ yearly degradation rate is included as an inset

$\lambda(t)$ is calculated as the ratio of the failure probability density function to the reliability function:

$$
\lambda(t)=\frac{f(t)}{R(t)}
$$

The function $\lambda(t)$ is usually referred to as the bathtub curve in reliability literature precisely because of its typical shape. It usually shows an initial decrease corresponding to the infant mortality period; a second constant stretch corresponding to the mature period of life for the product; and eventually it starts to increase during the wear-out period. Our model only considers degradation-induced failure and thereby only focuses on the so-called wear-out period. Accordingly, the

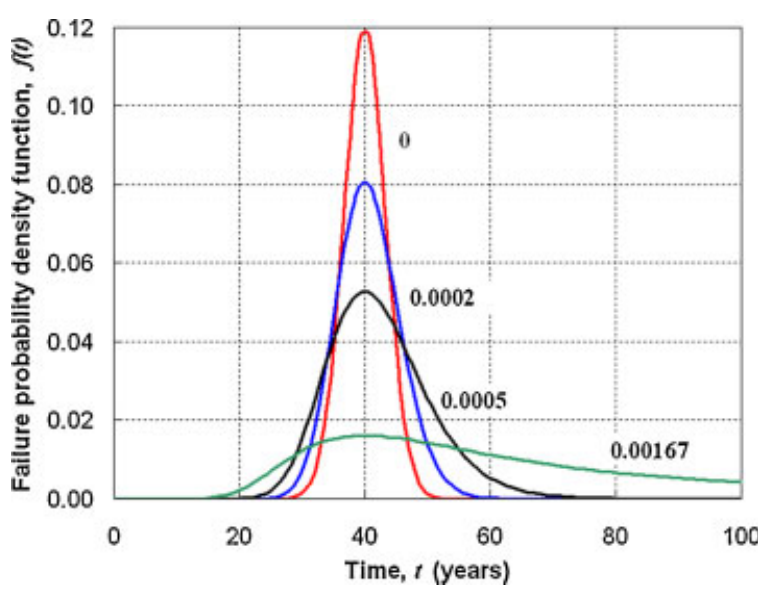

Figure 6. Failure probability density function for different $B / P_{0}$ values and a fixed $0.5 \%$ yearly degradation rate 


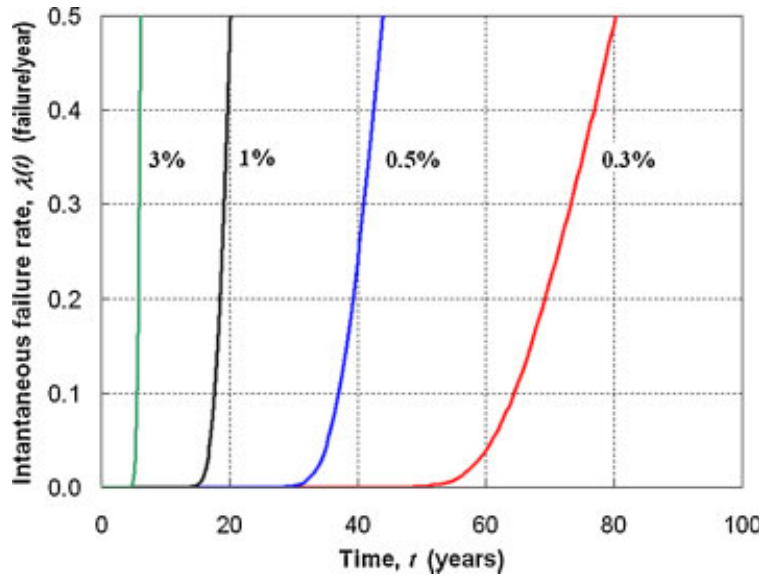

Figure 7. Instantaneous failure rate function $-\lambda(t)-$ for different yearly degradation rates and $B=0$

instantaneous failure rate function will be a monotonically increasing function as shown in Figures 7 and 8.

\section{Time parameters}

With this model it is also possible to evaluate different important time-related reliability data such as the MTTF, time to $50 \%$ reliability $\left(t_{50}\right)$ and power warranty time $\left(t_{\mathrm{W}}\right)$ :

- MTTF has been defined as the mean (average) time to failure of a system or product. This value is very common in electronic devices, which usually have a constant failure rate. However, in our case the failure rate is not constant (as previously discussed) and the

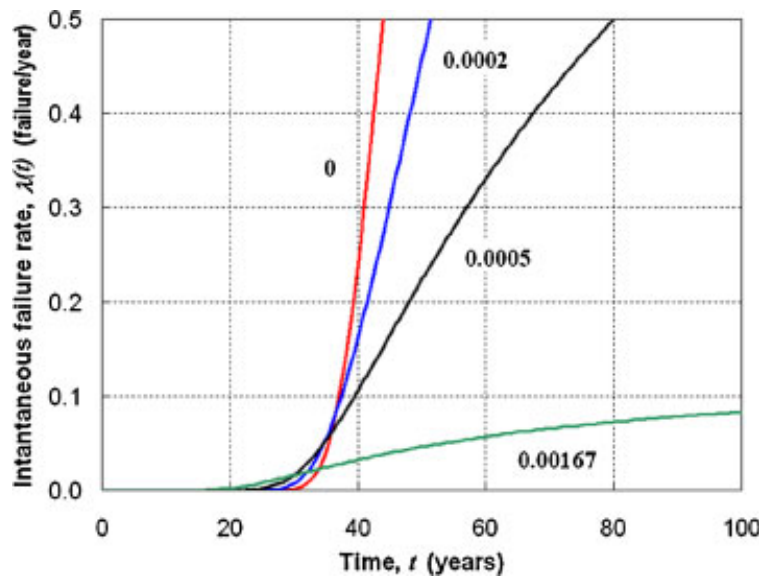

Figure 8. Instantaneous failure rate function $-\lambda(t)$ - for different $B / P_{0}$ values and $0.5 \%$ yearly degradation rate
MTTF has to be evaluated as the mean of the failure probability density function (Equation 10):

$$
\operatorname{MTTF}=\int_{0}^{\infty} t f(t) \mathrm{d} t
$$

- $t_{50}$ or the time at which $50 \%$ of the PV modules have failed can be evaluated simply using this model. It can be seen in Figure 2 that $t_{50}$ occurs when the average power coincides with the power limit (i.e. $P_{\text {limit }}-\mu(t)$ is equal to zero) or, equivalently, when the reliability function equals $0.5\left(R\left(t_{50}\right)=0 \cdot 5\right)$. Therefore, using Equation 3 it follows that:

$$
t_{50}=\frac{P_{0}-P_{\text {limit }}}{A}
$$

- Power warranty time $\left(t_{\mathrm{W}}\right)$ can be evaluated by taking into account the percentage of returned solar modules that a manufacturer can accept. If we assume that every failed module is going to be detected and sent back to the manufacturer then the probability that a module has failed at a given instant coincides with the percentage of failed (returned) modules and equals $1-R(t)$. Therefore, power warranty times $\left(t_{\mathrm{W}}\right)$ can be assessed using the following expression:

$$
K \leq 1-R\left(t_{\mathrm{W}}\right)
$$

where $K$ is the maximum percentage of returns that a manufacturer can withstand.

In summary, the MTTF and $t_{50}$ are related to situations where reliability is much less than it should be during the useful life of a PV module. Accordingly, though widely used in reliability analysis of other products, they are not very useful parameters in order to set reasonable power warranty times. In other words, given a MTTF or a $t_{50}$ there is nothing we can tell about $t_{\mathrm{W}}$. In general, the overall picture for the reliability analysis can only be assessed using the reliability functions.

\section{MODEL APPLICATION}

In this section we will apply the reliability model presented in Section 'Model Development' to PV modules. To do so, values must be given to the parameters involved in the reliability functions (i.e. those in Equations 9-14). We will use the limited experimental records available, summarized in Section 
'Review of Field Degradation Studies of PV Modules', to gather these data.

In order to simplify the analysis, we shall assume that the power limit that defines failure is $80 \%$ of the nominal power of the modules as this is a usual figure in PV module warranties $\left(P_{\text {limit }}=0 \cdot 8 P_{0}\right)$. It has been shown in Section 'Product Warranty and Qualification Tests' that some manufacturers refer to this limit as the minimum nominal power by taking power tolerances and even measurement tolerances into account. Though the calculations involved are uncomplicated (Equation 1), we will not do so here in order to not obscure the main goal of the calculations. In summary, we will refer the failure criterion to the average nominal power, $P_{0}$.

The value of $\sigma_{0}$ can be related to $P_{0}$ using the concept of manufacturing tolerance for the module output power. This tolerance is usually established in such a way that it spans $\pm 3 \sigma$ either side of the mean value. It is known that in a Gaussian probability density function approximately $99.7 \%$ of the data are within $3 \sigma$ of the mean, so using this criterion, $99.7 \%$ of the PV modules produced would meet the power ratings. Accordingly, the relationship between $\sigma_{0}, P_{0}$ and the manufacturing tolerance for the module output power $(\gamma)$ is given by the expression:

$$
P_{0}-3 \sigma_{0}=P_{0}(1-\gamma)
$$

From Equation 15, it follows directly that:

$$
\frac{\sigma_{0}}{P_{0}}=\frac{\gamma}{3}
$$

In the following calculations we will consider a value for the manufacturing tolerance for the module output power of $\pm 5 \%$ (very common), which using Equation 16 yields a value of 0.0167 for the ratio $\sigma_{0} / P_{0}$.

As summarized in Section 'Review of Field Degradation Studies of PV Modules', the value for the yearly degradation rate (i.e. the ratio $A / P_{0}$ ) varies between 0.003 and $0.03(0.3-3 \%$ yearly degradation). Within this interval a most likely value of $0.005(0.5 \%)$ will be considered, when needed.

Finally, to determine the applicable range for the ratio $B / P_{0}$ there is not much information available. In Reference 20 it was shown that the standard deviation of the module output power probability density function doubled after 10 years of field operation. It is straightforward to calculate that the associated value of $B / P_{0}$ equals 0.00167 . However, other sources ${ }^{26}$ report significantly lower values for the parameter $B$.
Thereby, in this analysis several cases for the ratio $B / P_{0}$ will be considered ranging from $B / P_{0}=0$ to $B / P_{0}=0 \cdot 00167$.

Finally, the tolerable amount of returned modules during the power warranty period has to be set (i.e. the value for $K$ in Equation 14). In the following, calculations, it will be assumed that during the power warranty period only $1 \%$ of PV modules can fail $(K=0 \cdot 01)$.

Once the parameters involved in the reliability functions are quantified we can proceed with their calculation.

\section{Reliability function for PV modules}

Figure 3 shows the reliability function for different values of the yearly degradation rate when $B / P_{0}=0$ (i.e. no timely increase of the standard distribution of the module power). The higher the yearly degradation rate, the earlier the roll off of the reliability occurs. For an $A / P_{0}$ greater than $1 \%$ this roll-off occurs clearly before 25 years of operating life, making it unreasonable to offer such warranties. From Figure 3, it seems that to offer power warranties of longer than 25 years the yearly degradation rate must be lower than $0.5 \%$.

Figure 4 shows the reliability function for a fixed yearly degradation rate of $0.5 \%$ and different values of the ratio $B / P_{0}$. This figure visualizes the great influence of $B / P_{0}$ in the reliability function, even for moderate yearly degradation rates. For the higher value of $B / P_{0}$ considered, ${ }^{20}$ a 25 -year power warranty period would be unreachable. As anticipated by Equation 13, all curves cross at $R=0.5$ (i.e. $t_{50}$ is the same for all since it does not depend on $B$ ). In a simple interpretation the higher the ratio $B / P_{0}$, the lower the slope at the cross point, $t_{50}$.

The precise quantification of the power warranty time by means of the reliability function will be addressed in Section 'Time Parameters', where the insets in Figures 3 and 4 will be discussed in detail.

\section{Failure probability density function for $P V$ modules}

Figure 5 plots the failure probability density function for the particular case when the standard deviation of the module power distribution does not increase over time $\left(B / P_{0}=0\right)$, for several yearly degradation rates. In such a case, this function follows a Gaussian probability density function; the mean value being at the time when $50 \%$ of the PV modules have failed $\left(t_{50}\right)$. 
As Figure 5 shows, the higher the value of the ratio A/ $P_{0}$, the earlier failures appear and the failures take place within a shorter period of time (i.e. the standard deviation of the failure probability density function is lower). This latter statement will be clarified when discussing the instantaneous failure rate function. Of course, information on power warranty times can also be derived from Figure 5, though in a more indirect manner. The inset in Figure 5 shows how $t_{\mathrm{W}}$ could be calculated just by integrating $f(t)$.

Figure 6 details the case when $\sigma$ increases over time for a fixed yearly degradation rate of $0.5 \%$. In this case the failure probability density function is asymmetric and hence does not fit a Gaussian distribution. This fact is not so clearly observed for moderate values of $B / P_{0}$ but is evident for high values of this parameter. The maximum of all the failure probability density functions in Figure 6 coincides with the median (not the mean) and occurs at $t_{50}$. Essentially, the higher the value of the ratio $B / P_{0}$ is, the earlier failures appear and the longer the failing period spans in time (i.e. the standard deviation of the failure probability density function increases). In other words, for high values of $B / P_{0}$ we lose reliability as a result of a prompt occurrence of a moderate amount of failures which extend for a prolonged time.

\section{Instantaneous failure rate function for PV modules}

Following the same philosophy as in previous sections Figure 7 details the instantaneous failure rate function for different yearly degradation rates and $B / P_{0}=0$, while Figure 8 represents the same function for a fixed yearly degradation rate of $0.5 \%$ and different values of the ratio $B / P_{0}$.

In Figures 7 and 8 it can be seen how the instantaneous failure rate function resulting from degradation increases over time starting at a very low failure rate during the first years of operation (lower than $10^{-4}$ failures per year), as expected for the wear-out period (see discussion in Section 'Main Reliability Functions). These failure rates are much lower than failure rate field data published in the literature, ${ }^{1-3}$ which are in the order of $10^{-3}$ failures per year. A first consequence of this discrepancy is that almost all failures reported occurring during the first years of operation must be catastrophic failures (not modelled by this instantaneous failure rate function) and not failures caused by degradation.

The failure rate attributable to an increase in degradation over time can be seen in Figure 7 for different yearly degradation rates and no increase in $\sigma$ over time $\left(B / P_{0}=0\right)$. Figure 7 shows that after several years, depending on the particular yearly degradation rate, the predominant failure mechanism is degradation and not catastrophic failures. In other words, after several years the degradation rate grows beyond the level of $10^{-3}$ failures per year, associated with catastrophic failures. The time at which degradation failures begin to dominate depends on the yearly degradation rate. These periods vary from a few years for the high yearly degradation rates (3\%), up to 50 years if yearly degradation rate is as low as $0.3 \%$. Figure 7 also shows that the lower the yearly degradation rate the later failures start occurring and the slope of the failure rate is reduced.

If the standard deviation increases over time, failures will appear earlier as can be seen in Figure 8 , but thereafter the slope of the failure rate is reduced.

\section{Time parameters}

Time at which reliability is $50 \%\left(t_{50}\right)$

The time at which reliability is 0.5 can be readily calculated using Equation 13. At this stage, the average power coincides with the power limit $\left(P_{\text {limit }}\right)$ as can be seen in Figure 2. Assuming again a $0.5 \%$ yearly degradation, it follows directly that $t_{50}$ equals 40 years (see Figure 1). Of course, the power warranty time must be much lower than this value because $50 \%$ of failures cannot be allowed during the warranty period. It is worth mentioning that the parameter $t_{50}$ only depends on the definition of failure $\left(P_{\text {limit }} / P_{0}\right)$ and on the yearly degradation rate $\left(A / P_{0}\right)$ and does not depend on how the standard deviation of the module output power evolves over time $\left(B / P_{0}\right)$.

\section{MTTF}

As discussed in Section 'Model Development', the MTTF can be calculated using Equation 12. Based on this equation we can conclude that for the particular case that the failure probability function is a Gaussian function $\left(B / P_{0}=0\right)$; the MTTF is equal to $t_{50}$. On the other hand, if $B / P_{0} \neq 0$ the failure probability function is asymmetric and the MTTF (the mean of the function $f(t)$ ) value is slightly higher than $t_{50}$ (the median of the function $f(t)$ ). Therefore, in no case is the MTTF a reasonable reference to set power warranty times.

Finally, it should be considered that this model only takes into account the failures resulting from the 
degradation of the PV modules. If both degradation and catastrophic failures are to be considered, the resulting MTTF value will be lower than the MTTF evaluated only for degradation failures.

\section{Warranty time $\left(t_{W}\right)$}

The warranty time is a commercial value that depends basically on reliability during the warranty period. Reliability during the warranty period must be high enough to minimize the return of the product, in our case PV modules. In the inset in Figure 3, we have represented the reliability function (from 0.95 to 1 ) for different yearly degradation rates and no evolution of the standard deviation over time $\left(B / P_{0}=0\right)$. In that figure a horizontal line has been drawn indicating the minimum reliability bearable during the power warranty time (which is $99 \%$ for a $K=1 \%$ ). The intercept of this line with each reliability curve yields the corresponding reasonable value for the warranty time $\left(t_{\mathrm{W}}\right)$. The inset shows that only yearly degradation rates of less than $0.5 \%$ may offer power warranties of more than 25 years.

Similarly, in the inset in Figure 4 we have represented the reliability function (from 0.95 to 1 ) for a fixed $0.5 \%$ yearly degradation rate and different values of the ratio $B / P_{0}$. In this case the quantitative impact on the power warranty time of the parameter $B /$ $P_{0}$ can be assessed. Again, the intercept of the line of minimum reliability with each reliability curve yields the corresponding reasonable value for the warranty time $\left(t_{\mathrm{W}}\right)$. In this case only the highest value of $B / P_{0}$ fails to achieve a power warranty time of more than 25 years. Figure 4 makes the importance of the parameter $B / P_{0}$ evident; for the same moderate yearly degradation rate, the advisable power warranty time would vary from less than 20 years (for $B /$ $P_{0}=0.00167$ ) up to more than 30 years (for $B / P_{0}=0$ ).

\section{SUMMARY AND CONCLUSIONS}

Degradation models have demonstrated that they are very useful in evaluating the reliability of highly reliable products where degradation is the main failure mechanism, which is precisely the case of PV modules. We have developed a degradation model that predicts PV module reliability based on field degradation data. With the reliability model it is possible to evaluate the different reliability functions and through them it is straightforward to quantify some relevant time parameters realistically such as, for instance, the warranty time for the product.

In Section 'Product Warranty and Qualification Tests' it was shown that long-term warranties and qualification standards do not assure the reliability levels implicit in the extended power warranty times offered by most PV module manufacturers. Long-term warranties do not imply that a particular product is reliable, if they are not the outcome of thorough field tests of comparable duration. This is not definitely the case in PV modules, where indefensible warranties are sometimes offered because of the pressure on sales and hard competition. Issue that is worth considering in relation to PV module reliability and power warranties is that, up to now, failures resulting from degradation have not usually been taken into consideration because of the difficulties in measuring individual PV modules in a system. However, recently PV systems are changing from small isolated systems to large gridconnected power stations. In this new scenario the customers will be more sensitive to power losses and a reliability model based on degradation may become of utmost importance.

In Section 'Review of Field Degradation Studies of PV Modules', the scarce literature on PV module degradation field studies was summarized. The main conclusions from this comparative analysis are:

- The average power of a set of modules in field operation decreases linearly over time.

- The yearly degradation rate for crystalline silicon PV modules ranges from 0.3 to $3 \%$ and seems to be constant during the wear-out period of the product. During the first year, somewhat higher degradation rates were observed in some studies.

- The power distribution of the modules tends to broaden over time, i.e. the standard deviation of the power distribution increases over time.

Once these basic parameters from field experiments are known, the mathematical foundations of the model could be developed and applied to a practical case: the production of $\mathrm{PV}$ modules with nominal power $P_{0} \pm 5 \%$, with a maximum number of returns of $1 \%$ of the production, and with a power warranty that becomes effective when the module power reaches $80 \%$ of its nominal value. The main conclusions from this quantitative analysis were:

- PV manufacturers must analyse the PV module power degradation carefully before setting the 
power warranty times. The optimum power warranty time strongly depends on power degradation rates and time-dependent power variability. With this model it is possible to evaluate the power warranty times by means of measuring or estimating a few key parameters that rule the PV module power degradation.

- From the reliability function it can be concluded that the yearly degradation rate must be less than $0.5 \%$ in order to provide the present 25 -year power warranties or longer. If the power module standard deviation increases significantly over time still lower yearly degradation rates will be necessary.

- The failure distribution function is a normal distribution if the standard deviation is constant $(B=0)$. It is asymmetric when the standard deviation increases over time $(B \neq 0)$.

- The instantaneous failure rate due to degradation increases over time, is very low $\left(<10^{-4}\right.$ failures per year) during the first few years. This means that at the beginning of the product's operational life the most common failure mechanism is catastrophic failure, while thereafter degradation will take over as the main failure mechanism. Failures resulting from degradation start to appear after a few years (if the yearly degradation is $3 \%$ ) or up to 50 years (if the yearly degradation is as low as $0.3 \%$ ). If the standard deviation increases over time the failures tend to appear earlier but at a lower rate.

- MTTF values for crystalline silicon PV modules derived from degradation calculations are in the range of several tens of years and hence have values significantly lower than those published in the literature. This discrepancy supports the fact that MTTF values reported thus far are the result of catastrophic failures during the first few years of operation and do not take degradation into account.

In summary, what has been presented in this paper is a rigorous approach to obtaining relevant and defensible power warranties, and other reliability data, based on measured degradation rates and timedependent power variability. In the derivation of the model some assumptions have been made about future behaviour-i.e. linear degradation rates-although the approach can be used for other assumed functional profiles as well. The approach documented in this paper explicitly shows manufacturers how to make reasonable and sensible warranty projections.

\section{Acknowledgements}

This paper has been supported by the Spanish Ministry for Education and Science with research project with reference TEC2005-02745.

\section{REFERENCES}

1. Maish A. Defining requirements for improved photovoltaic system reliability. Progress in Photovoltaics: Research and Applications 1999; 7: 165-173.

2. Wohlgemuth JH, Cunningham DW, Nguyen AM, Miller J. Long term reliability of PV module. 20th European Photovoltaic Solar Energy Conference, Barcelona, Spain, 2005; 1942-1946.

3. Woghgemuth JH. Long term photovoltaic module reliability. NCPV and Solar Program Review Meeting, 2003.

4. Coit DW, Evans JL, Vogt NT, Thomson JR. A method for correlating field life degradation with relaibility prediction for electronic modules. Quality and Reliability Engineering International 2005; 21: 715-726.

5. Cereghetti N, Burá E, Chianesse D, Frisen G, Realini A, Rezzonico S. Power and energy production PV modules statistical considerations of 10 years of activity. $3 r d$ Conference on Photovoltaic Energy Conversion, Osaka, Japan, May 11-18 2003.

6. International Electrotechnical Commission (IEC) 61215: 2nd edn, 2005. Crystalline silicon terrestrial photovoltaic modules-Design qualification and type approval.

7. Bishop J, Ossenbrink H. Results of five years of module qualification testing to CEC specification 503. 25th PVSC 1996; pp. 1191-1196.

8. Ossenbrink H, Simple T. Results of 12 years of module qualification to the IEC 61215 standard and CEC specification 503. Proceedings on 3rd World Conference on Photovoltaic Energy Conversion, 2003; pp. 1882-1887.

9. Wohlgemuth J. Reliability testing of PV modules 1st WCPEC, Hawaii, USA, 5-9 December 1994; pp. 889-892.

10. Chianese D, Realini A, Cereghetti N, Rezzonico S, Burá E, Friesen G, Bernasconi A. Analysis of weathered c-Si PV modules. 3rd World Conference on Photovoltaic Energy Conversion, Osaka, Japan, 2003; pp. 2922-2926.

11. Realini A, Bura E, Cereghetti N, Chianese D, Rezzonico $\mathrm{S}$. Mean time before failure of photovoltaic modules (MTBF-PVm), Annual Report 2002-Swiss Federal Office of Energy, 2002.

12. Blanquet D, Boulanger $P$, Montgareuil AG, Jourde $P$, Mattera F. Advances needed in standardization of PV components and systems. 3rd World Conference Photovoltaic Energy Conversion, 2003; 1877-1881.

13. Varadi PF, Domínguez R, Schaeffer L. Quality management in photovoltaics: quality control training manual 
for manufacturers. http://www.worldbank.org/astae/ qpp/PVGAP/pvgap.htm

14. Swanson RM. A vision for crystalline silicon photovoltaics. Progress in Photovoltaics: Research and Applications 2006; 14: 443-453.

15. Carr AJ, Pryor TL. A comparison of the performance of different PV module types in temperate climates. Solar Energy 2004; 76: 285-294.

16. Meyer EL, Dyck EE. Assessing the reliability and degradation of photovoltaic module performance parameters. IEEE Transactions on Reliability 2004; 53(1): 83-92.

17. Quintana MA, King DL, McMahon TJ, Osterwald RC. Commonly observed degradation in field-aged photovoltaic modules. 29th Photovoltaic Specialists Conference, 2002; 1436-1439.

18. Sakamoto S, Oshiro T. Dominant degradation of crystalline silicon photovoltaic modules manufactures in 1990́s. 20th European Photovoltaic Solar Energy Conference, Barcelona, 2005; pp. 2155-2158.

19. Osterwald CR, Benner JP, Pruett J, Anderberg A, Rummeland S, Ottoson L. Degradation in weathered crystalline-silicon PV modules apparently caused by UV radiation. 3rd World Conference on Photovoltaic Energy Conversion, Osaka, Japan, 2003; pp. 2911-2915.

20. Reis AM, Coleman NT, Marshall MW, Lehman PA, Chamberlain CE. Comparison of PV module performance before and after 11-years of field exposure. Proceedings of the 29th IEEE Photovoltaic Specialists Conference, New Orleans, Louisiana, USA, 2002.

21. Muirhead IJ, Hawkins BK. An assessment of photovoltaic power in the Telstra network. Solar 95.

22. Raghuraman B, Laksman V, Kuitche J, Shisler W, Tamizhani G, Kapoor H. An overview of SMUDs outdoor photovoltaic test program at Arizona State University. 2006 IEEE 4th IEEE World Conference on Photovoltaic Energy Conversion, Hawaii, USA, 2006.

23. Marion B, Adelstein J. Long-term performance of the SERF PV systems. NCPV and Solar Program Review Meeting, 2003.

24. Dunlop ED, Halton D. The performance of crystalline silicon photovoltaic solar modules after 22 years of continous outdoor esposure. Progress in Photovoltaics: Research and Applications 2006; 14: 53-64.

25. Quintana MA, King DL, Hosking FM, Kratovich JA, Johnson RW, Hansen BR, Dhere NG, Pandit MB. Diagnostic analysis of silicon photovoltaic modules after 20-year field exposure. 28th IEEE Photovoltaic Specialist Conference, Anchorage, AL, 2000.
26. Berman D, Faiman D. EVA browning and the time dependence of $\mathrm{I}-\mathrm{V}$ curve parameters on PV modules with and without mirror-enhancement in a desert environment. Solar Energy Materials and Solar Cells 1997; 45: 401-412.

27. Morita K, Inoue $\mathrm{T}$, Kato $\mathrm{T}$, Tsuda I, Hishikawa $\mathrm{Y}$. Degradation factor analysis of crystalline-Si PV modules through long-term field exposure test. $3 \mathrm{rd}$ World Conference on Photovoltaic Energy Conversion, Osaka, Japan, 2003; pp. 1948-1951.

28. Realini A, Burá E, Cereghetti N, Chianese D, Rezzonico S. Study of 20-year old PV plant (MTBF Project). 17th EPVSEC, Munich, Germany, 2001.

29. Huang W, Askin RG. Reliability analysis of electronic devices with multiple competing failure modes involving performance aging degradation. Quality and Reliability Engineering International 19: 241-254.

30. Crk V. Reliability assessment from degradation data. 2000 Proceedings Annual Reliability and Maintainability Symposium, 2000; 155-161.

31. Ribeiro Branco de Oliveira V, Colosito EA. Comparison of methods to estimate the time-to-failure distribution in degradation tests. Quality and Reliability Engineering International 2004; 20: 363-373.

32. Joo Bae S, Kuo W, Kvam PH. Degradation models and lifetime distributions. Reliability Engineering and System Safety 2007; 92: 601-608.

33. Sakamoto S, Oshiro T. Field test results on the stability of crystalline silicon photovoltaic modules manufactured in the 1990́s. 3rd World Conference on Photovoltaic Energy Conversion, Osaka, 2003; pp. 1888-1891.

34. Berman D, Biryukov S, Faiman D. EVA laminate browning after 5 years in a grid-connected. Mirror assisted, photovoltaic system in the Negev desert: effect on module efficiency. Solar Energy Material and Solar Cells 1995; 36: 421-422.

35. Green MA. Silicon photovoltaic modules: a brief history of the first 50 years. Progress in Photovoltaics: Research and Applications 2005; 13: 447-455.

36. Xie J, Pecht M. Reliability prediction modelling of semiconductor light emitting device. IEEE Transactions on Device and Materials Reliability 2003; 3: 218222.

37. Chuang S, Ishibashi A, Kijima S, Nakayama N, Ukita M, Taniguchi S. Kinetic model for degradation of lightemitting diodes. IEEE Journal of Quantum electronics 1997; 33: 970-979.

38. Connor PO. Practical Reliability Engineering, 4th edn, John Wiley \& Sons: New York 2002. 